\title{
THE IDENTIFIABILITY PROBLEM FOR REPAIRABLE SYSTEMS SUBJECT TO COMPETING RISKS
}

\author{
TIM BEDFORD, ${ }^{*}$ Strathclyde University
}

BO H LINDQVIST, ${ }^{* *}$ Norwegian University of Science and Technology

\begin{abstract}
Within reliability theory, identifiability problems arise through competing risks. If we have a series system of several components, and if that system is replaced or repaired to as good as new on failure, then the different component failures represent competing risks for the system. It is well known that the underlying component failure distributions cannot be estimated from the observable data (failure time and identity of failed component) without nontestable assumptions such as independence.

In practice many systems are not subject to the "as good as new" repair regime. Hence the objective of this paper is to contrast the identifiability issues arising for different repair regimes.

We consider the problem of identifying a model within a given class of probabilistic models for the system. Different models corresponding to different repair strategies are considered: a partial repair model where only the failing component is repaired; perfect repair, where all components are as good as new after a failure; and minimal repair, where components are only minimally repaired at failures. We show that on the basis of observing a single socket, the partial repair model is identifiable, while the perfect and minimal repair models are not.

Keywords: Competing risks; Identifiability; Reliability; Marked point process; Ergodicity; Markov chain; Joint survival distribution
\end{abstract}

\footnotetext{
* Postal address: Department of Management Science, Strathclyde University, Graham Hills Building, 40 George Street, Glasgow, Scotland. Email: tim@mansci.strath.ac.uk

** Postal address: Department of Mathematical Sciences, Norwegian University of Science and Technology, N-7491 Trondheim, Norway. Email: bo@math.ntnu.no
} 
2000 Mathematics Subject Classification: Primary 60J27, 90B25

Secondary 60G35, 37A30, 60K10

\section{Introduction and basic model}

Consider a series system with $n$ components, denoted $C_{1}, C_{2}, \ldots, C_{n}$. Let the system be observed from time $t=0$ and suppose that upon each failure the system is immediately repaired (in some way to be specified) and put into operation. Denote the successive failure times by $T_{1}, T_{2}, \ldots$. Assume that at each system failure we can identify a unique failing component. Thus we observe a marked point process $\left(T_{j}, Z_{j}\right)$ where $Z_{j}=i$ if component $C_{i}$ fails at time $T_{j}$. At failures $T_{j}$ the components are repaired or replaced according to given repair strategies. Various such strategies will be considered.

Since the ideas are well described in the case of two components, we shall let $n=2$ in most of our discussion.

We shall be concerned with observations arising from a single system or socket. This means that we observe part of a single realisation of a marked point process. When all the components are repaired to as good as new on the failure of a single component then we actually have a renewal process. A single realisation of the marked point process gives rise to an infinite number of realisations of the renewal process. This does not enable us to identify the joint distribution of the component lifetimes, or even the marginal distributions, in general (see $[2,1])$. The reason for this is that only partial information about the joint distribution is observable. Only by making nontestable assumptions about the model class we are dealing with are we able to identify the marginals or the joint distribution. The classical approach to competing risks is to assume that the competing risks are statistically independent of each other, in which case we have identifiability as discussed in $[2,1]$. More generally it is sometimes possible to restrict the class of joint distributions under consideration so that the problem becomes identifiable, but this is very application specific.

In the context of reliability, the assumption of a renewal process is a fairly strong assumption. (In many cases it is plausible, for example in high reliability systems where the cost of unscheduled maintenance is high, such as in the nuclear industry. In other situations it is more questionable). The question then arises: do we still have 
identifiability problems when the renewal assumption does not hold? The purpose of this paper is to discuss this question. To make a comparison, we contrast the renewal model with two weaker models: A partial repair model where the failed component is replaced or repaired to as good as new at failures (while the other components are not repaired), and (weaker still) minimal repair of the failed component.

We shall see that, in contrast to the renewal model, the partial repair assumption enables us to identify the underlying model. The weaker model, supposing just minimal repair of the failed component, is however again not identifiable.

This last fact can briefly be explained as follows. It is well known for the single component case that if failures are minimally repaired, then the resulting failure time process is a nonhomogeneous Poisson process. In the case of several components which are minimally repaired, it follows in a similar way that the failure processes of different components must be independent nonhomogeneous Poisson processes. The observed marked point process can thus give no information about dependence of the components. Moreover, since we observe only one Poisson process for each component, we are not even able to identify their intensity functions from a single realisation of the point process.

\subsection{Definition}

Consider now the case involving two components. The components are assumed to have basic failure rates $\lambda_{1}(t), \lambda_{2}(t)$, respectively, but when working within the system, the lifetimes of the two components are coupled. This is modelled by assuming that the conditional failure intensity of component $C_{1}$ given the history is

$$
\nu_{1}\left(s_{1}, s_{2}\right)=\lambda_{1}\left(s_{1}\right)+\lambda_{1 *}\left(s_{1}, s_{2}\right)
$$

where $s_{1}, s_{2}$ are the ages of the components $C_{1}$ and $C_{2}$, respectively. We shall mostly think of the age of a component as the time since last replacement. Thus $\lambda_{1 *}\left(s_{1}, s_{2}\right)$ can be viewed as the additional failure intensity of component $C_{1}$ arising from component $C_{2}$, and is a function of the current age of $C_{2}$, and possibly of the age of $C_{1}$.

In the same manner we define a function $\lambda_{2 *}$ so that $\lambda_{2 *}\left(s_{1}, s_{2}\right)$ is the additional failure intensity of component $C_{2}$ due to component $C_{1}$. Thus the conditional failure 
intensity of component $C_{2}$ given the history is

$$
\nu_{2}\left(s_{1}, s_{2}\right)=\lambda_{2}\left(s_{2}\right)+\lambda_{2 *}\left(s_{1}, s_{2}\right)
$$

In general we assume that $\lambda_{1}(t), \lambda_{2}(t)$ are continuous nonnegative functions, and that the $\lambda_{1 *}, \lambda_{2 *}$ are such that (1) and (2) are both continuous and nonnegative. In addition we need to make the uniqueness assumptions

$$
\begin{aligned}
& \lambda_{1 *}(u, 0)=0 \text { for all } u \\
& \lambda_{2 *}(0, u)=0 \text { for all } u
\end{aligned}
$$

These conditions mean that the additional failure intensity of component $C_{1}$ is 0 when component $C_{2}$ is new, and vice versa.

Note that (3-4) ensure that any pair of nonnegative functions $\nu_{1}\left(s_{1}, s_{2}\right), \nu_{2}\left(s_{1}, s_{2}\right)$ can be uniquely put in the form (1-2), although $\lambda_{1 *}$ and $\lambda_{2 *}$ might take negative values.

Note also that the components are independent if and only if $\lambda_{1 *}\left(t_{1}, t_{2}\right)=\lambda_{2 *}\left(t_{1}, t_{2}\right) \equiv$ 0 . This is because independence must mean that $\lambda_{1 *}\left(t_{1}, t_{2}\right)\left(\lambda_{2 *}\left(t_{1}, t_{2}\right)\right)$ is constant in $t_{2}\left(t_{1}\right)$ and hence must equal 0 by $(3-4)$.

We remark that the additive forms in (1-2) could be replaced by a multiplicative form if we replaced 0 by 1 (or some known number) in conditions (3-4). The results of the paper would still hold. It is a matter of taste as to which version is used: We like the interpretation of $\lambda_{i}$ as the intrinsic failure rate of the component, and of $\lambda_{i *}$ as the additional failure rate induced by the other components of the system.

\subsection{Identifiability}

When we talk about identifiability of the model we mean that on the basis of a single realization of the marked point process we can determine the functions $\lambda_{1}, \lambda_{2}$, $\lambda_{1 *}$, and $\lambda_{2 *}$.

We conclude this section with a remark about the assumptions (3-4). These assumptions are necessary for identifiability in this model, for otherwise we can only determine the sums

$$
\lambda_{1}(s)+\lambda_{1 *}(s, 0) \text { and } \lambda_{2}(s)+\lambda_{2 *}(0, s)
$$


Specification of the ratio's

$$
\frac{\lambda_{1 *}(s, 0)}{\lambda_{1}(s)} \text { and } \frac{\lambda_{2 *}(0, s)}{\lambda_{2}(s)}
$$

would be sufficient to obtain identifiability in general (clearly (3-4) imply that the ratio's equal zero). Such numbers, which we call moduli of identifiability, could be estimated by expert judgement, but cannot be identified from the observations we consider in this paper.

\section{A partial repair model}

Assume that when the system fails, the failing component is replaced by a new component of the same kind, while the non-failing component is not repaired nor maintained. The natural data arising in this model consists of a sequence $\left(t_{n}, z_{n}\right)$ indicating the time of the $n$th failure $t_{n}$ and the number of the failed component $z_{n}$.

We show that, under reasonable conditions, the natural data generated by a single sequence of observations enables us to identify the model. This is done by showing that the underlying marked point process is uniquely ergodic. We shall use some results from [6], and will first recall the notation used there.

The canonical space of simple marked point processes is the space

$$
M_{1}=\left\{\psi=\left\{\left(t_{n}, z_{n}\right): n \geq 0\right\}\right\}
$$

where $t_{n}$ is the time of the $n$th event and $z_{n}$ is its mark. The space is endowed with the product topology and can be metrized to become a complete separable metric space. The collection of Borel sets is denoted $\mathcal{B}\left(M_{1}\right)$. Usually we will consider $M_{1}$ to be embedded within the set of two-sided sequences

$$
M_{2}=\left\{\psi=\left\{\left(t_{n}, z_{n}\right): n \in \mathbb{Z}\right\}\right\}
$$

which can similarly be considered a complete separable metric space. We also consider subsets corresponding to simple marked point processes for which the origin is occupied,

$$
\begin{aligned}
& M_{1, o}=\left\{\psi=\left\{\left(t_{n}, z_{n}\right): n \geq 0, t_{0}=0\right\}\right\} \\
& M_{2, o}=\left\{\psi=\left\{\left(t_{n}, z_{n}\right): n \in \mathbb{Z}_{+}, t_{0}=0\right\}\right\} .
\end{aligned}
$$


The counting process associated with $M_{1}$ is the mapping $N(t)(\psi)$ that gives the number of events minus one for the marked point process $\psi$ in the interval $[0, t]$. Thus in our application where the first event is at time 0 , the counting process will count the number of failures, rather than the number of "repairs".

We consider two different mappings on $M_{1}$; they may also be defined on $M_{2}$ in the obvious way. The first is the time shift $\theta_{s}$ that simply subtracts time $s$ from the event times,

$$
\theta_{s}(\psi)=\left\{\left(t_{N(s)+n+1}-s, z_{N(s)+n+1}\right): N(s)+n+1 \geq 0\right\} .
$$

The second mapping is the event time shift $\theta_{(j)}$ which shifts the origin to the $j$ th event of the marked point process,

$$
\theta_{(j)}(\psi)=\theta_{t_{j}}(\psi)
$$

A distribution $\mathbf{P}$ on $M_{1}$ is time stationary if for all $s \geq 0$ and Borel sets $\mathcal{E} \in \mathcal{B}\left(M_{1}\right)$,

$$
\mathbf{P}\left(\theta_{s}(\psi) \in \mathcal{E}\right)=\mathbf{P}(\psi \in \mathcal{E})
$$

We say that $\mathbf{P}$ on $M_{1}$ is event stationary if for all $j \in \mathbb{Z}_{+}$and Borel sets $\mathcal{E} \in \mathcal{B}\left(M_{1}\right)$,

$$
\mathbf{P}\left(\theta_{(j)}(\psi) \in \mathcal{E}\right)=\mathbf{P}(\psi \in \mathcal{E}) .
$$

Similar definitions hold for distributions on $M_{2}$.

Now, a given distribution $\mathbf{P}$ need not be time or event stationary, but one can consider two averaging processes corresponding to averaging $\mathbf{P}$ over time shifts and averaging over event shifts to produce stationary distributions. The distributions $\mathbf{P}^{0}$ and $\mathbf{P}^{*}$ are defined by the following limits (when the limits exist):

$$
\begin{aligned}
\mathbf{P}^{0}(\psi \in \mathcal{E}) & =\lim _{n \rightarrow \infty} \frac{1}{n} \sum_{j=1}^{n} \mathbf{P}\left(\theta_{(j)}(\psi) \in \mathcal{E}\right) ; \\
\mathbf{P}^{*}(\psi \in \mathcal{E}) & =\lim _{t \rightarrow \infty} \frac{1}{t} \int_{0}^{t} \mathbf{P}\left(\theta_{s}(\psi) \in \mathcal{E}\right) d s .
\end{aligned}
$$

The distributions $\mathbf{P}^{0}$ and $\mathbf{P}^{*}$ are called the empirical distributions. Note for the moment that these distributions are not based on single sample paths. Below we shall show that in our case the limits exist for $\mathbf{P}$ concentrated on just a single ("typical") point. To do this we need the concept of ergodicity. 
A distribution $\mathbf{P}$ is said to be ergodic under a transformation if the only invariant Borel sets are trivial, that is, for any Borel set $\mathcal{E}$ that is preserved under the transformation one has $\mathbf{P}(\mathcal{E})$ equal to zero or one.

From a practical point of view it is interesting to know about the time-stationary distribution of the marked point process. For the purposes of proving identifiability it will however be easier to consider event-stationary distributions. This is because we can construct a Markov chain representing the event dynamics of the system. Afterwards we can derive information about the time-stationary distribution if needed.

\subsection{Continuous state discrete time Markov chain representation}

In the above discussion we have used the canonical representation of a marked point process. An alternative representation uses the interarrival times instead of the absolute times. Yet another representation, that will be convenient for us is in terms of a continuous state, discrete time Markov chain. This representation makes the underlying structure of the process clearer and enables us to use the theory of continuous state Markov chains to check ergodicity.

Recall that the intensity functions only depend on the ages of the components. Hence the state of the system can be represented by a pair $\left(s_{1}, s_{2}\right)$ of non-negative numbers. As time increases, both components age at the same rate, but eventually one of them fails leading to its replacement and the age being reset to 0. Depending on which component fails, the system either follows path 1 or path 2 in Figure 1.

Note that if we had $n$ components instead of 2 then the state space would be the non-negative orthant of $\mathbb{R}^{n}$.

Since the behaviour between failures is deterministic we can consider the process just at the failure times, i.e. when it lies on the boundary of the non-negative quadrant. As demonstrated in the next subsection, this process can be represented in a particularly simple manner when $n=2$.

2.1.1. Obtaining a Markov chain from the marked point process. The behaviour of the system at time $t$ is independent of the past given the times $s_{1}, s_{2}$ since last replacement for each component. Thus we can define the following Markov chain $\left\{X_{n}\right\}$ where $n$ is failure number. 


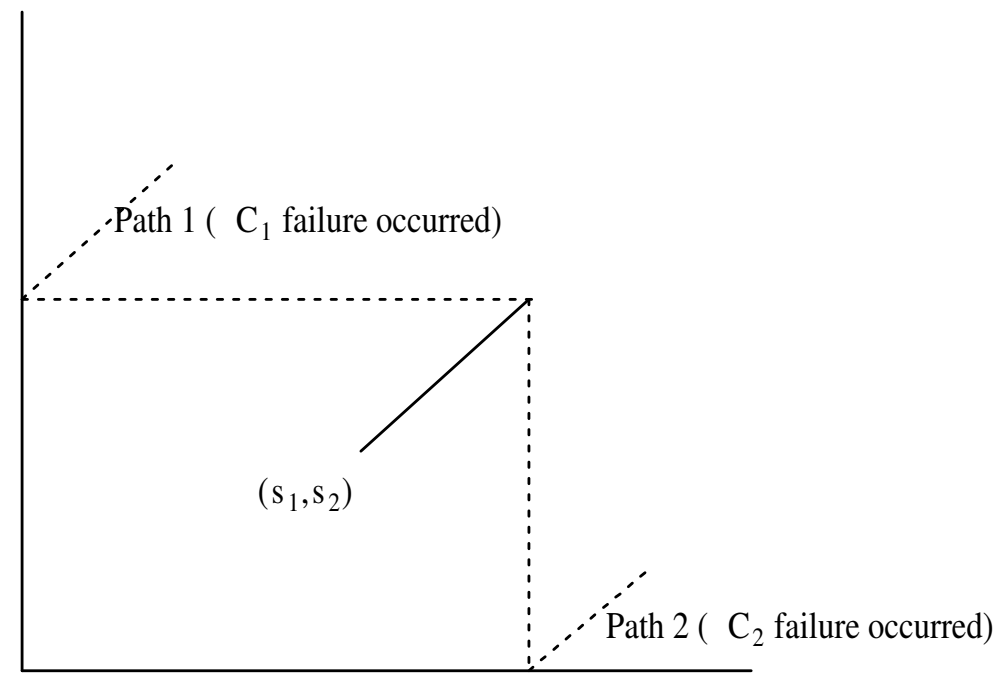

Figure 1: Time dependent evolution of the system

If the $n$th failure is a $C_{1}$-failure, and the time since the last $C_{2}$ failure is $s_{2}$, then put

$$
X_{n}=s_{2}
$$

If, on the other hand, the $n$th failure is a $C_{2}$-failure, and the time since the last $C_{1}$ failure is $s_{1}$, then we put

$$
X_{n}=-s_{1}
$$

The state space of the chain is therefore the set $S=\mathbb{R}$ (this effectively takes the boundary of the non-negative quadrant and flattens it out). Thus $X_{n}>0$ means that the $n$th failing component is $C_{1}$ and that the time since the last failure of $C_{2}$ is $X_{n}$. Conversely, $X_{n}<0$ means that the $n$th failing component is $C_{2}$ and that the time since the last failure of $C_{1}$ is $-X_{n}$. We write the mark of the $n$th component as

$$
m\left(X_{n}\right)= \begin{cases}1 & \text { if } X_{n}>0 \\ 2 & \text { if } X_{n}<0\end{cases}
$$

The case $X_{n}=0$ means that both components failed simultaneously. Under our assumptions this occurs with zero probability, except possibly as a starting condition.

Now suppose $X_{n}=s_{2}>0$. Then the $n$th failure was a $C_{1}$-failure, and $X_{n+1}$ is either $s_{2}+t$ or $-t$ for some $t>0$. The former case occurs if the next failure is another 
$C_{1}$-failure, occurring a time $t$ after the previous failure, while the latter corresponds to a $C_{2}$-failure occurring a time $t$ after the previous failure.

2.1.2. Obtaining the marked point process from the Markov chain. A realization $\left(X_{n} ; n \geq\right.$ 0 ) of the Markov chain together with the time $t_{0}$ of event 0 specifies the realization of the marked point process as follows:

1. Event 0 occurs at time $t_{0}$, and is a failure of component $m\left(X_{0}\right)$.

2. If $\operatorname{sgn}\left(X_{1}\right) \neq \operatorname{sgn}\left(X_{0}\right)$ then Event 1 occurs at time $t_{1}=t_{0}+\left|X_{1}\right|$ and is a failure of component $m\left(X_{1}\right)$.

3. If $\operatorname{sgn}\left(\mathrm{X}_{1}\right)=\operatorname{sgn}\left(\mathrm{X}_{0}\right)$ then Event 1 occurs at time $t_{1}=t_{0}+\left|X_{1}\right|-\left|X_{0}\right|$ and is a failure of component $m\left(X_{1}\right)$.

4. ...

5. If $\operatorname{sgn}\left(\mathrm{X}_{\mathrm{n}}\right) \neq \operatorname{sgn}\left(\mathrm{X}_{\mathrm{n}-1}\right)$ then Event $n$ occurs at time $t_{n}=t_{n-1}+\left|X_{n}\right|$ and is a failure of component $m\left(X_{n}\right)$.

6. If $\operatorname{sgn}\left(X_{n}\right)=\operatorname{sgn}\left(X_{n-1}\right)$ then Event $n$ occurs at time

$$
t_{n}=t_{n-1}+\left|X_{n}\right|-\left|X_{n-1}\right|
$$

and is a failure of component $m\left(X_{n}\right)$.

2.1.3. Transition densities and intensities of the marked point process. Given the relation between the marked point process and Markov chain representations, we should expect that the intensity and transition densities can be written in terms of one another.

In general the transition densities of a Markov chain $X_{n}$ on $S$ with the same allowed moves as the described chain, are determined by a set of subdensities for $t>0$, associated with the different possible moves,

$$
\begin{array}{lll}
f_{11}(t \mid s) \text { for transitions from } & s>0 & \text { to } s+t>0 \\
f_{12}(t \mid s) \text { for transitions from } & s>0 & \text { to }-t<0 \\
f_{21}(t \mid s) \text { for transitions from } & s<0 & \text { to } t>0 \\
f_{22}(t \mid s) \text { for transitions from } & s<0 & \text { to } s-t<0
\end{array}
$$




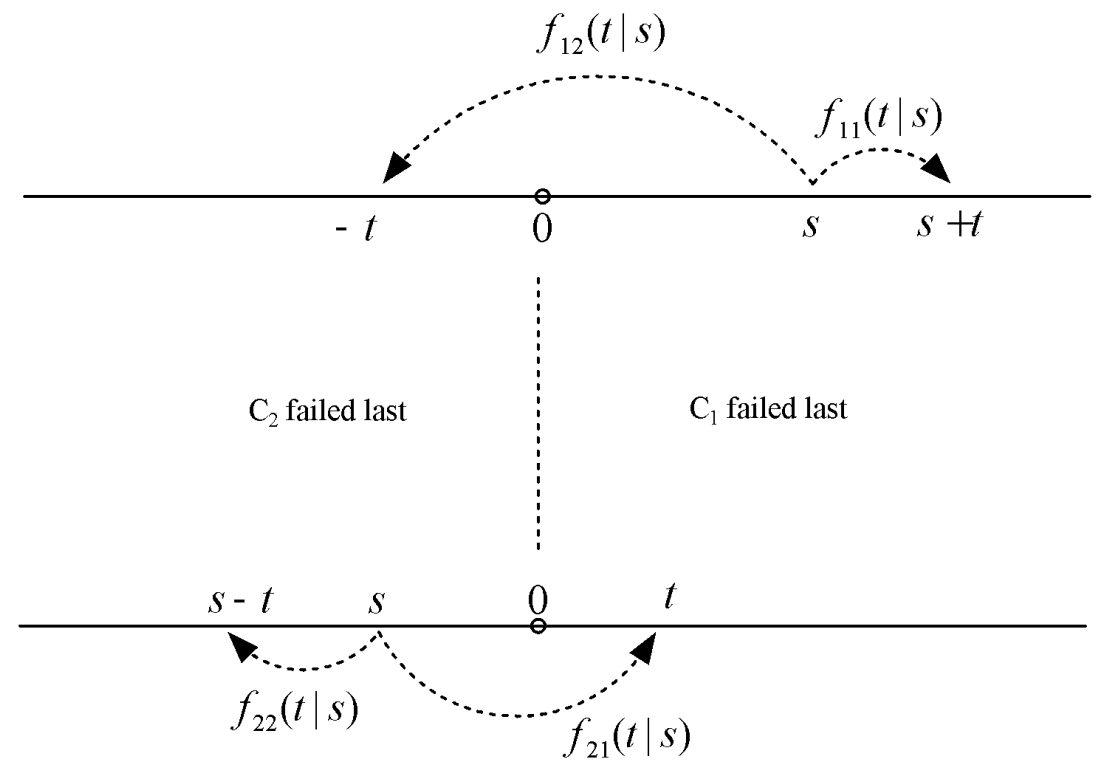

Figure 2: Allowable transitions and densities

Note the meaning of the indices of the $f$-densities, for example the index 12 of $f_{12}(t \mid s)$ means that we go from a $C_{1}$-failure (1) to a $C_{2}$-failure (2) and that the starting state was $s$ (which is necessarily $>0$ ). Using the identification of $S$ with $\mathbb{R}$ we will then have the complete transition densities from state $s>0$ to be

$$
p(s, x)= \begin{cases}f_{11}(x-s \mid s) & \text { for } x>s \\ f_{12}(x \mid s) & \text { for } x<0\end{cases}
$$

(see Figure 2). A similar density can be written down for start in $s<0$, namely

$$
p(s, x)= \begin{cases}f_{21}(x \mid s) & \text { for } x>0 \\ f_{22}(s-x \mid s) & \text { for } x<s\end{cases}
$$

We remark that the sums

$$
\begin{aligned}
& f_{1 \cdot}(t \mid s)=f_{11}(t \mid s)+f_{12}(t \mid s) \\
& f_{2 \cdot}(t \mid s)=f_{21}(t \mid s)+f_{22}(t \mid s)
\end{aligned}
$$

are density functions for the elapsed time from the occurrence of failure number $n$ to the occurrence of failure number $n+1$, given $X_{n}=s$. 
Returning to the model formulation in Section 1.1 we can express the $f$-functions in terms of the functions $\nu_{1}, \nu_{2}$ as follows,

$$
\begin{aligned}
f_{11}(t \mid s)= & \nu_{1}(t, s+t) \exp \left\{-\int_{0}^{t}\left(\nu_{1}(u, s+u)+\nu_{2}(u, s+u)\right) d u\right\}, \\
& s>0, t>0 \\
f_{12}(t \mid s)= & \nu_{2}(t, s+t) \exp \left\{-\int_{0}^{t}\left(\nu_{1}(u, s+u)+\nu_{2}(u, s+u)\right) d u\right\}, \\
& s>0, t>0 \\
f_{21}(t \mid s)= & \nu_{1}(-s+t, t) \exp \left\{-\int_{0}^{t}\left(\nu_{1}(-s+u, u)+\nu_{2}(-s+u, u)\right) d u\right\}, \\
& s<0, t>0 \\
f_{22}(t \mid s)= & \nu_{2}(-s+t, t) \exp \left\{-\int_{0}^{t}\left(\nu_{1}(-s+u, u)+\nu_{2}(-s+u, u)\right) d u\right\}, \\
& s<0, t>0
\end{aligned}
$$

We now show

Theorem 1. For each possible set of the four functions (5-8) there exists a unique set of functions $\lambda_{1}, \lambda_{1 *}, \lambda_{2}, \lambda_{2 *}$ satisfying the requirements (1-4).

Proof. By uniqueness of the $\lambda_{1}, \lambda_{1 *}, \lambda_{2}, \lambda_{2 *}$ (Section 1.1) it is enough to show that (11-14) imply unique functions $\nu_{1}, \nu_{2}$. To prove this last fact, note that to any density function $f(t)$ of a continuous, positive random variable there exists a unique hazard rate $z(t)$ such that $f(t)=z(t) \exp \left\{-\int_{0}^{t} z(u) d u\right\}$. Hence if $f_{1}(t)$ and $f_{2}(t)$ are nonnegative functions with sum equal to the $f(t)$ above, then there exist unique $z_{1}(t)$ and $z_{2}(t)$ with sum $z(t)$ and such that $f_{i}(t)=z_{i}(t) \exp \left\{-\int_{0}^{t} z(u) d u\right\}$ for $i=1,2$. The result follows from this.

It follows that if the observation of the process $\left\{X_{n}\right\}$ from time 0 to infinity determines all transition densities, then the partial repair model is identifiable by a single sequence. This leads us to the investigation of ergodicity of the chain.

\subsection{Ergodicity of the Markov chain}

We will demonstrate ergodicity of the chain by applying results from Doob [3]. The basic assumption of Doob is his Condition D (known as Doeblin's condition): This says that there is a positive finite valued measure $\phi$ on the state space, an integer $k \geq 1$ 
and a positive $\epsilon$ such that for all $\xi$,

$$
p^{(k)}(\xi, A) \equiv P\left(X_{k} \in A \mid X_{0}=\xi\right) \leq 1-\epsilon, \text { if } \phi(A) \leq \epsilon
$$

Condition D does not automatically follow for the Markov chain we have defined. Extra assumptions are needed to show that it holds. The assumption we use is that the probability of failure of one component just after the other component has failed is bounded away from zero, independently of the age of the component. Formally, for each $\delta>0$ there exist $\eta=\eta(\delta)>0$ such that for all $s$,

$$
p(s,(-\delta, \delta))>\eta
$$

Proposition 1. Under condition (15) the Markov chain satisfies Condition D.

Proof. By the assumed continuity of the $\lambda$-functions, and the fact that there is an interval $C$ such that the probability of a transition to $C$ from 0 is positive, we conclude that there is $\eta^{\prime}>0$ and an interval $B \subset C$ such that the transition density to $b \in B$ from any $s \in(-\delta, \delta)$ has value at least $\eta^{\prime}$. By the assumption (15) we can then estimate the two-step transition density by

$$
p^{(2)}(s, b)>\eta^{\prime \prime}, \text { for all } b \in B, s \in \mathbb{R},
$$

for some $\eta^{\prime \prime}>0$. Hence for any Borel set $A \subset \mathbb{R}$, we can estimate the two step transition probability

$$
\begin{aligned}
p^{(2)}(s, A) & =1-p^{(2)}\left(s, A^{c}\right) \leq 1-p^{(2)}(s, B \backslash A) \\
& <1-\eta^{\prime \prime} \phi(B \backslash A) \leq 1-\eta^{\prime \prime}(\phi(B)-\phi(A))
\end{aligned}
$$

where $\phi$ is Lebesgue-measure.

Taking

$$
\epsilon=\frac{\phi(B) \eta^{\prime \prime}}{1+\eta^{\prime \prime}}
$$

we have

$$
\begin{aligned}
p^{(2)}(s, A) & <1-\eta^{\prime \prime} \phi(B)+\eta^{\prime \prime} \phi(A)=1-\left(1+\eta^{\prime \prime}\right) \epsilon+\eta^{\prime \prime} \phi(A) \\
& <1-\left(1+\eta^{\prime \prime}\right) \epsilon+\eta^{\prime \prime} \epsilon=1-\epsilon,
\end{aligned}
$$

and we see that Condition D holds. 
The consequence of Condition D is that the state space contains a countable number of minimal invariant sets. Since in our case the transition density from $s$ is positive outside the interval between 0 and $s$, we conclude that there is a single invariant minimal set, equal to $\mathbb{R}$, the whole state space. As a corollary of Theorem 5.7 in [3] we conclude that there is a single stationary distribution, which is therefore also ergodic.

Hence for any starting point of the Markov chain, the chain will converge (in Cesaro sense) to the ergodic stationary distribution $\pi$. In particular, for any Borel set $A$, and for any starting point $X_{0}$,

$$
\lim _{n \rightarrow \infty} \frac{1}{n} \sum_{1}^{n} \mathbf{1}_{A}\left(X_{i}\right)=\pi(A) .
$$

Suppose now that $X_{0}$ has the stationary distribution. Then, for any Borel sets $A$ and $B$, we can consider the probability $q(A, B)=\pi\left(X_{0} \in A, X_{1} \in B\right)$. By considering the Markov chain $Y_{n}=\left(X_{n}, X_{n-1}\right)$ on the state space $S \times S$ which is also uniquely ergodic we see that probability $q(A, B)$ can be determined in the same way. For any starting point $Y_{0}$,

$$
\lim _{n \rightarrow \infty} \frac{1}{n} \sum_{1}^{n} \mathbf{1}_{(A, B)}\left(\left(X_{i}, X_{i+1}\right)\right)=q(A, B) .
$$

We can write $q(A, B)$ in terms of the stationary distribution and the transition probabilities by $q(A, B)=\int_{A} p(x, B) d \pi(x)$. Since the transition densities are continuous we find

$$
p(x, B)=\lim _{\epsilon \rightarrow 0} q((x-\epsilon, x+\epsilon), B) / \pi((x-\epsilon, x+\epsilon)) .
$$

Similarly, by taking $B$ to be a small interval around a point $y$, and letting its diameter go to zero, we obtain the transition density from $x$ to $y$.

We conclude that the transition densities of the Markov chain, and hence also the conditional intensities of the marked point process, are identifiable from a single sequence.

\subsection{Sufficient conditions for ergodicity}

In this subsection we consider sufficient criterions for condition (15) in terms of the $\lambda$-functions.

Proposition 2. Suppose for each given $\delta>0$ there is an $\epsilon=\epsilon(\delta) \leq \delta$ and positive 
numbers $A_{i}(\delta), B_{i}(\delta), C_{i}(\delta)(i=1,2)$ such that for all $s>0$,

$$
\begin{aligned}
& \frac{\lambda_{2}(s+t)+\lambda_{2 *}(t, s+t)}{\lambda_{1}(t)+\lambda_{1 *}(t, s+t)} \geq A_{1}(\delta)>0, \quad t \in[\delta-\epsilon, \delta] \\
& \frac{\lambda_{1}(s+t)+\lambda_{1 *}(s+t, t)}{\lambda_{2}(t)+\lambda_{2 *}(s+t, t)} \geq A_{2}(\delta)>0, \quad t \in[\delta-\epsilon, \delta]
\end{aligned}
$$

(iii) $\int_{0}^{\delta}\left[\lambda_{1}(u)+\lambda_{1 *}(u, s+u)+\lambda_{2}(s+u)+\lambda_{2 *}(u, s+u)\right] d u \leq B_{1}(\delta)<\infty$

(iv) $\quad \int_{0}^{\delta}\left[\lambda_{1}(s+u)+\lambda_{1 *}(s+u, u)+\lambda_{2}(u)+\lambda_{2 *}(s+u, u)\right] d u \leq B_{2}(\delta)<\infty$

$(v) \quad \int_{\delta-\epsilon}^{\delta}\left[\lambda_{1}(u)+\lambda_{1 *}(u, s+u)+\lambda_{2}(s+u)+\lambda_{2 *}(u, s+u)\right] d u \geq C_{1}(\delta)>0$

(vi) $\int_{\delta-\epsilon}^{\delta}\left[\lambda_{1}(s+u)+\lambda_{1 *}(s+u, u)+\lambda_{2}(u)+\lambda_{2 *}(s+u, u)\right] d u \geq C_{2}(\delta)>0$

Then the conditon (15) holds.

Proof. Let $s>0$ and $\delta>0$ be arbitrarily chosen. Then with $\epsilon=\epsilon(\delta)$ we get from (i) that

$$
\lambda_{2}(s+t)+\lambda_{2 *}(t, s+t) \geq \frac{A_{1}(\delta)}{1+A_{1}(\delta)}\left(\lambda_{1}(t)+\lambda_{1 *}(t, s+t)+\lambda_{2}(s+t)+\lambda_{2 *}(t, s+t)\right)
$$

for all $t \in[\delta-\epsilon, \delta]$, and hence

$$
\begin{aligned}
p(s,(- & \delta, \delta)) \\
& \geq \int_{0}^{\delta} f_{12}(t \mid s) d t \geq \int_{\delta-\epsilon}^{\delta} f_{12}(t \mid s) d t \\
& \geq \frac{A_{1}(\delta)}{1+A_{1}(\delta)} \exp \left\{-\int_{0}^{\delta-\epsilon}\left[\lambda_{1}(u)+\lambda_{1 *}(u, s+u)+\lambda_{2}(s+u)+\lambda_{2 *}(u, s+u)\right] d u\right\} \\
& \times\left(1-\exp \left\{-\int_{\delta-\epsilon}^{\delta}\left[\lambda_{1}(u)+\lambda_{1 *}(u, s+u)+\lambda_{2}(s+u)+\lambda_{2 *}(u, s+u)\right] d u\right\}\right) \\
& \geq \frac{A_{1}(\delta)}{1+A_{1}(\delta)} e^{-B_{1}(\delta)}\left(1-e^{-C_{1}(\delta)}\right) \equiv \eta(\delta)>0
\end{aligned}
$$

where we used (iii),(v) and the fact that

$$
\int_{a}^{b} z(t) \exp \left\{-\int_{0}^{t} z(u) d u\right\} d t=\exp \left\{-\int_{0}^{a} z(u) d u\right\}\left(1-\exp \left\{-\int_{a}^{b} z(u) d u\right\}\right)
$$

for any function $z(\cdot)$. Thus condition (15) holds when $s>0$.

The proof of (15) for the case $s<0$ is similar.

Remark. Conditions (i)-(vi) of Proposition 2 follow trivially if all the involved $\lambda$ functions are nonnegative and uniformly bounded away from 0 and $\infty$. In practice, however, we need to handle cases with unbounded functions as well. Indeed, the most 
commonly used parametric models for failure intensities are in fact unbounded. The following corollary covers many models of practical interest.

Corollary 1. Suppose $\lambda_{1}(t), \lambda_{2}(t)>0$ for all $t>0$ and suppose these two functions are bounded away from 0 as $t \rightarrow \infty$. Suppose next that $\lambda_{1 *}(s, t) \equiv \rho_{1}(t) \geq 0$ is a nonnegative function depending only on $t$, and that similarly $\lambda_{2 *}(s, t) \equiv \rho_{2}(s) \geq 0$ is a nonnegative function depending only on s. Suppose also that the integrals $\int_{0}^{\delta} \lambda_{i}(u) d u$, $\int_{0}^{\delta} \rho_{i}(u) d u(i=1,2)$ are finite for all $\delta>0$.

Then the two conditions

$$
\begin{aligned}
\liminf _{s \rightarrow \infty} \frac{\lambda_{2}(s)}{\rho_{1}(s)} & \equiv L_{1}>0 \\
\liminf _{s \rightarrow \infty} \frac{\lambda_{1}(s)}{\rho_{2}(s)} & \equiv L_{2}>0
\end{aligned}
$$

(where we put $f(s) / g(s)=\infty$ if $g(s)=0$ ) together imply the assumptions of Proposition 2.

Proof. Consider first (i) of Proposition 2. If $\rho_{1}(\cdot)$ is bounded, then (i) holds since $\lambda_{2}(s)$ by assumption is bounded away from 0 as $s \rightarrow \infty$. If $\rho_{1}(\cdot)$ is not bounded, then (16) readily implies that $\liminf _{s \rightarrow \infty} \frac{\lambda_{2}(s+t)+\rho_{2}(t)}{\lambda_{1}(t)+\rho_{1}(s+t)}=L_{1}$ for all $t>0$ from which (i) follows. Now (ii) follows similarly from (17). The remaining conditions (iii)-(vi) are simple consequences of the assumptions.

Remark. Note that the conditions of the corollary include cases where possibly $\lambda_{i}(0)=0$ or $\lim _{t \rightarrow 0} \lambda_{i}(t)=\infty$. Conditions (16-17) intuitively state that the influence of a component's age on the failure intensity of the other component can be of no larger order of magnitude than the influence of its age on its own basic intensity. Theoretically, this has to do with the fulfillment of Condition D, but in practice it also seems to be a reasonable assumption in the partial repair model.

2.3.1. A Weibull type partial repair model. In analyses of repairable systems one commonly uses failure intensities of the power-law form, $\gamma \beta t^{\beta-1}$, where $\gamma, \beta>0$. This gives a Weibull renewal process in the case of perfect repairs, and a so-called Weibull nonhomogenous Poisson process in the minimal repair case. Here we suggest a Weibull 
partial repair model as follows, using the notation from Corollary 1:

$$
\begin{aligned}
\lambda_{i}(t) & =\gamma_{i} \beta_{i} t^{\beta_{i}-1} \\
\rho_{i}(t) & =\kappa_{i} \mu_{i} t^{\mu_{i}-1}
\end{aligned}
$$

for $i=1,2$, and all parameters assumed positive.

It is seen that the assumptions of Corollary 1 are satisfied when $\beta_{1} \geq \mu_{2}$ and $\beta_{2} \geq \mu_{1}$. In words these state that the exponent of the age parameter $t$ should be no larger for the influence of age on the other component, than on the component itself.

2.3.2. Models with bounded dependence terms. A generalized exponential model. As already noted, one should expect that in practice the influence of a component on the failure intensity of another component is considerably less than the basic failure intensity of the component itself. For certain applications it might even seem reasonable to assume that the functions $\lambda_{i *}(s, t)$ are bounded. For practical modelling the following parametric functions could then be tried,

$$
\begin{aligned}
& \lambda_{1 *}(s, t)=\gamma_{1}\left(1-e^{-\kappa_{1} s}\right)\left(1-e^{-\mu_{1} t}\right) \\
& \lambda_{2 *}(s, t)=\gamma_{2}\left(1-e^{-\kappa_{2} t}\right)\left(1-e^{-\mu_{2} s}\right)
\end{aligned}
$$

Here the $\mu_{i}$ are parameters determining the influence of the other component, while the $\kappa_{i}$ can be considered as tuning parameters for the interdependence between the ages of the components. Note that $\kappa_{1}=\infty\left(\kappa_{2}=\infty\right)$ leads to the case where $\lambda_{1 *}(s, t)$ $\left(\lambda_{2 *}(s, t)\right)$ is a function of $t(s)$ alone.

A simple model involving the constant functions $\lambda_{1}(t) \equiv \lambda_{1}, \lambda_{2}(t) \equiv \lambda_{2}$, and dependence terms given as in (18-19) is likely to be useful for situations where one suspects a dependence between basically exponential lifetimes.

\subsection{The stationary distribution of the point process}

In the above section we have shown that the conditional intensities of the marked point process are identifiable using the stationary distribution of a related process. We now show how this is related to the stationary distribution of the point process.

We showed above that an infinite sequence from the state space $S$ of the Markov chain, together with a time for the zeroth event, specify an point from the canonical 
space of the marked point process. If we restrict our attention to these marked point processes for which the origin is occupied then we see that we have a bijection $\alpha: S^{\mathbb{Z}_{+}} \rightarrow$ $M_{1, o}$ (or $\alpha: S^{\mathbb{Z}} \rightarrow M_{2, o}$ if we want to consider bi-infinite processes). Furthermore, the Markov shift commutes with the event shift $\theta_{(1)}$,

$$
\begin{array}{ccc}
S^{\mathbb{Z}_{+}} & \overrightarrow{\theta_{(1)}} & S^{\mathbb{Z}_{+}} \\
\alpha \downarrow & & \downarrow \alpha \\
M_{1} & \overrightarrow{\theta_{(1)}} & M_{1} .
\end{array}
$$

Hence the unique stationary ergodic distribution that we have constructed for the Markov chain corresponds (via $\alpha$ ) to a unique stationary ergodic distribution for the event shift operator. Following [6] we call this event shift stationary distribution $\mathbf{P}^{0}$.

Now, from the theory of marked point processes (in [6]) we know how to construct a time stationary distribution from an event stationary distribution: essentially we just have to put in the information about the distance to the origin of the 0th event, and reweight the event stationary distribution to take account of the size, $T_{0}$, of the interval between the 0 th and 1 st events. We paraphrase Theorem 2.9 and Propsition 2.8 of [6]:

Theorem 2. If $0<E_{\mathbf{P}^{0}}\left(T_{0}\right)<\infty$ then an ergodic time stationary distribution $\mathbf{P}^{*}$ exists satisfying

$$
\begin{aligned}
\mathbf{P}^{*}(\mathcal{E}) & =\lim _{t \rightarrow \infty} \frac{1}{t} \int_{0}^{t} \mathbf{P}^{0}\left(\theta_{s}(\psi) \in \mathcal{E}\right) d s \\
& =\frac{E_{\mathbf{P}^{0}}\left(\int_{0}^{T_{0}} \mathbf{1}\left\{\theta_{s}(\psi) \in \mathcal{E}\right\} d s\right)}{E_{\mathbf{P}^{0}}\left(T_{0}\right)} .
\end{aligned}
$$

Note that Sigman states this result for general stationary distributions (not necessarily ergodic) and also gives a formula describing $\mathbf{P}^{0}$ in terms of $\mathbf{P}^{*}$.

It is easy to see that if the density functions given in (9-10) have uniformly bounded first moment then $0<E_{\mathbf{P}^{0}}\left(T_{0}\right)<\infty$ holds.

\subsection{Generalization to more than two components}

As we stated in the introduction, the main ideas can be discussed when we have a system involving just two components. The ideas can, however, be generalized to $n$ components. In this section we discuss briefly the way in which that can be done.

The first important step is to consider the conditional failure intensity of component 
$i$. This is written as

$$
\nu_{i}\left(s_{1}, \ldots, s_{n}\right)=\lambda_{i}\left(s_{i}\right)+\lambda_{i *}\left(s_{1}, \ldots, s_{n}\right),
$$

where $\lambda_{i}\left(s_{i}\right)$ is the failure rate of component $i$ at age $s_{i}, \lambda_{i *}\left(s_{1}, \ldots, s_{n}\right)$ is the additional failure intensity due to the influence of the other components at their respective ages $s_{1}, \ldots, s_{n}$. Again, we make the assumption that when the "other" components are new there is no additional influence on the failure intensity of component $i$,

$$
\lambda_{i *}\left(s_{1}, \ldots, s_{n}\right)=0
$$

for all $s_{i}$ when $s_{j}=0(j \neq i)$. Note that this includes the case where the failure intensity of each component $(i)$ is influenced additively by the other components, that is where $\lambda_{i *}\left(s_{1}, \ldots, s_{n}\right)=\sum_{j \neq i} \lambda_{i j}\left(s_{i}, s_{j}\right)$, and $\lambda_{i j}\left(s_{i}, s_{j}\right)=0$ if $s_{j}=0$.

The marked point process in this case is the same as before, except that the marking set is $\{1, \ldots, n\}$. Similarly, the state space for the continuous state Markov chain is the boundary of the non-negative quadrant of $\mathbb{R}^{n}$, where the vector elements represent the current age of each component. The faces of this boundary correspond to the sets of states where one given component failed last.

Expressions similar to those of (11-14) hold, that describe the likelihoods of transitions from one face of the state space to another. The natural generalization of Theorem 1 holds, so that if we are able to estimate the transition densities from observations, then we can also estimate the intensity functions.

Recalling that our aim is to show that under appropriate conditions we have identifiability of the intensity functions, we first show that, essentially, it just depends on whether or not the Markov chain is ergodic.

Theorem 3. Suppose that the Markov chain constructed above has a unique ergodic invariant probability distribution. Then the transition density functions of the Markov chain can be estimated (almost surely) from a single sequence of observations and the failure intensities of the competing risk model are identifiable.

We remark that even if the Markov chain did not have an ergodic invariant probability distribution, as long as there was a $\sigma$-finite ergodic distribution, the Hopf ratio ergodic theorem would still allow us to derive the transition density functions. Such considerations take us, however, away from the main point of the paper. 
We have given conditions for $n=2$ under which the Markov chain has a unique ergodic invariant probability distribution. It seems rather difficult to state very general conditions under which this holds when $n>2$ (or indeed optimal conditions when $n=2)$.

\section{Other repair models}

The standard models used for standard repairable systems with only one failure mode are renewal processes and nonhomogeneous Poisson processes. These correspond to, respectively, perfect and minimal repair at failures. In this section we consider the corresponding cases for the competing risk situation of the present paper.

\subsection{Perfect repair}

In this case both components are replaced at each system failure $T_{1}, T_{2}, \ldots$ It follows that the conditional failure intensities for the two components are, respectively,

$$
\begin{aligned}
& \lambda_{1}(t)+\lambda_{1 *}(t, t) \\
& \lambda_{2}(t)+\lambda_{2 *}(t, t)
\end{aligned}
$$

where $t$ is the time since last system failure. Thus $T_{1}, T_{2}, \ldots$ is a renewal process where the interarrival distribution is determined by the hazard rate

$$
\lambda_{1}(t)+\lambda_{1 *}(t, t)+\lambda_{2}(t)+\lambda_{2 *}(t, t)
$$

while the marks $Z_{1}, Z_{2}, \ldots$ have the distribution

$$
P\left(Z_{j}=1 \mid T_{j}-T_{j-1}=t\right)=\frac{\lambda_{1}(t)+\lambda_{1 *}(t, t)}{\lambda_{1}(t)+\lambda_{1 *}(t, t)+\lambda_{2}(t)+\lambda_{2 *}(t, t)}
$$

Since a single realisation of the marked point process leads to an infinite number of interarrival times, we conclude that the two functions $t \mapsto \lambda_{1}(t)+\lambda_{1 *}(t, t)$ and $t \mapsto \lambda_{2}(t)+\lambda_{2 *}(t, t)$ are identifiable. However, this is clearly not sufficient to recover the underlying functions $\lambda_{i}(\cdot), \lambda_{i}^{*}(\cdot, \cdot), i=1,2$. 


\subsection{Minimal repair}

Minimal repair means here that the age of each component is identical to the running time $t$. Thus the failure intensity for the two components at time $t$ are, respectively,

$$
\begin{aligned}
& \lambda_{1}(t)+\lambda_{1 *}(t, t) \\
& \lambda_{2}(t)+\lambda_{2 *}(t, t)
\end{aligned}
$$

Since the failure processes are completely independent of the (failure) history, and each has a deterministic conditional intensity function, it follows that the failure processes of $C_{1}$ and $C_{2}$ are stochastically independent nonhomogenous Poisson processes with intensity functions given by (24-25).

It should be noted that although (24-25) are identical to (22-23), we are in the minimal repair case not able to identify even these functions in general. This is because we have only one realisation of each of the two nonhomogeneous Poisson processes.

\section{Representation by a joint survival distribution}

Repair models involving a single component may often be described in terms of the survival function $R(t)$ for the lifetime of a new component (see e.g. Dorado et al. [4]). The question now arises: can the partial repair model described in the present paper be represented by a joint survival function, or is it more general? The purpose of this section is to explore this issue. Let $R\left(t_{1}, t_{2}\right)$ be a joint survival distribution, say for the pair $Y_{1}, Y_{2}$ of lifetimes. This means that $R$ is nonincreasing in each argument, and that $R\left(s_{1}, s_{2}\right)-R\left(t_{1}, s_{2}\right)-R\left(s_{1}, t_{2}\right)+R\left(t_{1}, t_{2}\right) \geq 0$ for all $s_{1} \leq t_{1}, s_{2} \leq t_{2}$. Further we assume that $R(0,0)=1$ and $R(\infty, \infty)=0$. We shall use $R(\cdot, \cdot)$ to define the probabilistic behaviour of the system and then relate it to the functions $\lambda_{1}, \lambda_{1 *}, \lambda_{2}, \lambda_{2 *}$.

Suppose the ages of the two components are $s_{1}, s_{2}$, respectively. We then consider the conditional distribution of $Y_{1}, Y_{2}$ given $Y_{1}>s_{1}, Y_{2}>s_{2}$, which has the joint survival distribution

$$
R_{\left(s_{1}, s_{2}\right)}\left(t_{1}, t_{2}\right)=\frac{R\left(s_{1}+t_{1}, s_{2}+t_{2}\right)}{R\left(s_{1}, s_{2}\right)}
$$

Let $T_{1}^{*}, T_{2}^{*}$ be a pair of lifetimes with this distribution. Then we let the time to next 
failure be $T^{*}=\min \left\{T_{1}^{*}, T_{2}^{*}\right\}$, which has density function

$$
\begin{aligned}
-(\partial / \partial t) R_{\left(s_{1}, s_{2}\right)}(t, t) & =\frac{-(\partial / \partial t) R\left(s_{1}+t, s_{2}+t\right)}{R\left(s_{1}, s_{2}\right)} \\
& =\frac{-D_{1} R\left(s_{1}+t, s_{2}+t\right)-D_{2} R\left(s_{1}+t, s_{2}+t\right)}{R\left(s_{1}, s_{2}\right)}
\end{aligned}
$$

where $D_{1}, D_{2}$ are differential operators with respect to the first and second component of the succeeding function, respectively.

Moreover, given that $T^{*}=t$, the probability of having $T^{*}=T_{i}^{*}(i=1,2)$ is seen to be

$$
\frac{D_{i} R\left(s_{1}+t, s_{2}+t\right)}{D_{1} R\left(s_{1}+t, s_{2}+t\right)+D_{2} R\left(s_{1}+t, s_{2}+t\right)}
$$

Thus, starting with components with ages $s_{1}, s_{2}$, the time to next failure has density (27), while (28) is the conditional probability that the failing component is $C_{i}$, given the failure time. Using the notation of our basic model it follows that

$$
\begin{aligned}
& \lambda_{1}\left(s_{1}+t\right)+\lambda_{1 *}\left(s_{1}+t, s_{2}+t\right)=-D_{1} R\left(s_{1}+t, s_{2}+t\right) / R\left(s_{1}+t, s_{2}+t\right) \\
& \lambda_{2}\left(s_{2}+t\right)+\lambda_{2 *}\left(s_{1}+t, s_{2}+t\right)=-D_{2} R\left(s_{1}+t, s_{2}+t\right) / R\left(s_{1}+t, s_{2}+t\right)
\end{aligned}
$$

Putting, respectively, $t=0, s_{2}=0$ in (29) and $t=0, s_{1}=0$ in (30) we get

$$
\begin{aligned}
& \lambda_{1}\left(s_{1}\right)=-D_{1} R\left(s_{1}, 0\right) / R\left(s_{1}, 0\right) \\
& \lambda_{2}\left(s_{2}\right)=-D_{2} R\left(0, s_{2}\right) / R\left(0, s_{2}\right)
\end{aligned}
$$

Together with (29-30) these equations determine the functions $\lambda_{1}, \lambda_{1 *}, \lambda_{2}, \lambda_{2 *}$ corresponding to a given joint survival function $R$.

Conversely, suppose instead that the functions $\lambda_{1}, \lambda_{1 *}, \lambda_{2}, \lambda_{2 *}$ are given and we want to recover $R(\cdot, \cdot)$, if possible. Then we can first solve the equations (31-32) to get

$$
\begin{aligned}
& R\left(s_{1}, 0\right)=\exp \left\{-\int_{0}^{s_{1}} \lambda_{1}(u) d u\right\} \\
& R\left(0, s_{2}\right)=\exp \left\{-\int_{0}^{s_{2}} \lambda_{2}(u) d u\right\}
\end{aligned}
$$

Next, by adding the equations (29-30) we get the equation

$$
\begin{aligned}
& -(\partial / \partial t) R\left(s_{1}+t, s_{2}+t\right) / R\left(s_{1}+t, s_{2}+t\right) \\
& \quad=\lambda_{1}\left(s_{1}+t\right)+\lambda_{1 *}\left(s_{1}+t, s_{2}+t\right)+\lambda_{2}\left(s_{2}+t\right)+\lambda_{2 *}\left(s_{1}+t, s_{2}+t\right)
\end{aligned}
$$


which by fixing $s_{1}, s_{2}$ implies that

$$
\begin{aligned}
& R\left(s_{1}+t, s_{2}+t\right)=R\left(s_{1}, s_{2}\right) \\
& \quad \times \exp \left\{-\left(\int_{0}^{t} \lambda_{1}\left(s_{1}+u\right) d u+\int_{0}^{t} \lambda_{2}\left(s_{2}+u\right) d u\right.\right. \\
& \left.\left.\quad+\int_{0}^{t} \lambda_{1 *}\left(s_{1}+u, s_{2}+u\right) d u+\int_{0}^{t} \lambda_{2 *}\left(s_{1}+u, s_{2}+u\right) d u\right)\right\}
\end{aligned}
$$

By putting, respectively, $s_{1}=0$ and $s_{2}=0$ in (36) we finally get the representation

$$
\begin{gathered}
R\left(t_{1}, t_{2}\right)=\exp \left\{-\left(\int_{0}^{t_{1}} \lambda_{1}(u) d u+\int_{0}^{t_{2}} \lambda_{2}(u) d u+\int_{0}^{t_{1}} \lambda_{1 *}\left(u, u+t_{2}-t_{1}\right) d u\right.\right. \\
\left.\left.+\int_{0}^{t_{1}} \lambda_{2 *}\left(u, u+t_{2}-t_{1}\right) d u\right)\right\}, t_{1}<t_{2} \\
R\left(t_{1}, t_{2}\right)=\exp \left\{-\left(\int_{0}^{t_{1}} \lambda_{1}(u) d u+\int_{0}^{t_{2}} \lambda_{2}(u) d u+\int_{0}^{t_{2}} \lambda_{1 *}\left(u+t_{1}-t_{2}, u\right) d u\right.\right. \\
\left.\left.+\int_{0}^{t_{2}} \lambda_{2 *}\left(u+t_{1}-t_{2}, u\right) d u\right)\right\}, t_{1}>t_{2}
\end{gathered}
$$

So far we conclude that if there is a solution $R(\cdot, \cdot)$ to the problem, then it is given by (37-38). On the other hand, if (37-38) is a solution, then (29-30) should be satisfied. Now substituting (37-38) in (29-30) shows that for consistency the following two conditions need to hold,

$$
\begin{aligned}
& \lambda_{2 *}\left(t, t+s_{2}\right)=\int_{0}^{t} D_{2} \lambda_{1 *}\left(u, u+s_{2}\right) d u+\int_{0}^{t} D_{2} \lambda_{2 *}\left(u, u+s_{2}\right) d u \\
& \lambda_{1 *}\left(t+s_{1}, t\right)=\int_{0}^{t} D_{1} \lambda_{1 *}\left(s_{1}+u, u\right) d u+\int_{0}^{t} D_{1} \lambda_{2 *}\left(s_{1}+u, u\right) d u
\end{aligned}
$$

It seems difficult to give these conditions an intuitive meaning. The following observation is interesting, however, in view of the models suggested earlier: If $\lambda_{1 *}\left(t_{1}, t_{2}\right)$ is a function of $t_{2}$ only, and $\lambda_{2 *}\left(t_{1}, t_{2}\right)$ is a function of $t_{1}$ only, then $\lambda_{1 *}\left(t_{1}, t_{2}\right)=\delta t_{2}$, $\lambda_{2 *}\left(t_{1}, t_{2}\right)=\delta t_{1}$ for some $\delta$ is the only possible solution to (39-40). This rules out the possibility of having survival distribution representations of the partial repair model in general. The following example illustrates this point in a simple case.

Example: Gumbel's bivariate exponential distribution. Gumbel [5] studies three different types of bivariate exponential distributions. One of these is given by the joint survival function

$$
R\left(t_{1}, t_{2}\right)=\exp \left\{-\left(\alpha t_{1}+\beta t_{2}+\delta t_{1} t_{2}\right)\right\}
$$


A straightforward computation using (29-32) leads to $\lambda_{1}(t)=\alpha, \lambda_{2}(t)=\beta, \lambda_{1 *}\left(t_{1}, t_{2}\right)=$ $\delta t_{2}, \lambda_{2 *}\left(t_{1}, t_{2}\right)=\delta t_{1}$, and these functions are easily verified to satisfy (39-40).

A more general model results if we change the definition of $\lambda_{1 *}$ to read $\lambda_{1 *}\left(t_{1}, t_{2}\right)=$ $\gamma t_{2}$ with the possibility of having $\gamma \neq \delta$. It follows from the above, however, that this model cannot be represented by a joint survival distribution, thus giving a simple example of a partial repair model which is not representable by a joint survival distribution.

It should be noted that neither of conditions (i) and (ii) of Proposition 2 are satisfied for the Gumbel model presented in this example. In fact, it may be checked directly that neither does Condition D hold here. Our conjecture, based on simulations from the model, is however that ergodicity still applies for this model. This indicates that Condition D can be an unnecessarily strong assumption for obtaining models of practical interest, a fact which turns out to be a general problem with the use of Condition D. Weaker conditions for ergodicity are therefore usually sought for.

\section{Conclusion}

We have shown that the identifiability issue becomes more complex when repair regimes other than perfect repair are used. Since, intuitively, the repair regime becomes weaker as we move from perfect repair through partial repair to minimal repair, one might imagine that the amount of information that can be derived about the components also decreases. However, this is not the case. The middle case actually gives full identifiability, while both extreme cases lack identifiability.

\section{References}

[1] Bedford T., And Meilijson, I. (1997), A characterisation of marginal distributions of (possibly dependent) stochastic variables which right-censor each other. Annals of Statistics, 25, 16221645 .

[2] Crowder, M. (2001), Classical Competing Risks, Chapman and Hall/CRC, Boca Raton.

[3] Doob, J. L. (1953), Stochastic Processes, John Wiley, New York.

[4] Dorado, C., Hollander, M. and Sethuraman, J. (1997). Nonparametric estimation for a general repair model. Annals of Statistics, 25, 1140-1160. 
[5] Gumbel, E. J. (1960), Bivariate exponential distributions. Journal of American Statistical Association, 55, 698-707.

[6] Sigman, K. (1995), Stationary marked point processes: an intuitive approach, Chapman and Hall, New York 